\title{
水合肼选择性还原炔烃至顺式烯烃研究
}

\author{
王金涛熊就凯李娟娟王佳利王克让* 李小六* \\ (河北大学化学与环境科学学院 药物化学与分子诊断教育部重点实验室 河北省化学生物学重点实验室
}

保定 071002)

\begin{abstract}
摘要 转移氢化反应是一种条件温和、反应转化率高的还原反应. 研究了一系列双烷基和双羟基修饰的炔类化合物, 在空气中水合肼的作用下选择性还原为顺式烯烃的方法, 其中水合肼作为转移氢化试剂, 空气中氧气作为氧化剂, 通 过氧化反应将肼氧化为二氮烯 $\mathrm{N}_{2} \mathrm{H}_{2}$ 分子, 进而二氮烯 $\mathrm{N}_{2} \mathrm{H}_{2}$ 分子选择性还原炔烃为顺式烯烃. 通过二维核磁共振谱证 明了生成的烯烃的构型为顺式结构.
\end{abstract}

关键词 转移氢化; 水合肼; 炔烃; 烯烃; 二氮烯

\section{Hydrogenation of Alkynes to cis-Alkenes with Hydrazine in Air}

\author{
Wang, Jintao Xiong, Jiukai Li, Juanjuan Wang, Jiali Wang, Kerang* Li, Xiaoliu* \\ (Key Laboratory of Medicinal Chemistry and Molecular Diagnosis of Ministry of Education, Key Laboratory of Chemical \\ Biology of Hebei Province, College of Chemistry and Environmental Science, Hebei University, Baoding 071002)
}

\begin{abstract}
Transfer hydrogenation is a mild and effective way in reduction reaction. In this paper, a simple approach of selectively hydrogenation of alkynes to cis-alkenes with $\mathrm{NH}_{2} \mathrm{NH}_{2} \cdot \mathrm{H}_{2} \mathrm{O}$ as a transfer hydrogenation agent in the presence of air without any catalysts or metals was developed. Furthermore, the configuration of cis-alkene was confirmed by the 2D NOESY spectrum.

Keywords transfer hydrogenation; hydrazine; alkynes; alkenes; diazene
\end{abstract}

氢化反应是有机合成反应中最重要的反应之一, 广 泛应用于精细化工和药物合成等领域 ${ }^{[1]}$, 氢化反应的氢 源一般为氢气. 近年来, 转移氢化反应 ${ }^{[2]}$ 引起了人们广 泛关注，具有反应条件温和、反应转化率高和不涉及高 压反应器等特点 ${ }^{[3]}$. 作为转移氢化试剂, 二氮烯 $\mathrm{N}_{2} \mathrm{H}_{2}$ (diazene) 分子在有机合成反应中的应用已经超过 100 年 ${ }^{[4]}$, 早在 1960 年代就发现其能选择性还原不饱和 $\mathrm{C}=$ $C$ 双键 ${ }^{[4]}$. 产生二氮烯 $\mathrm{N}_{2} \mathrm{H}_{2}$ 分子最直接的方法是水合肼 的氧化反应, 常用的氧化剂包括: $\mathrm{H}_{2} \mathrm{O}_{2} 、 \mathrm{NaIO}_{4}$ 、 $\mathrm{K}_{3}\left[(\mathrm{FeCN})_{6}\right]$ 和氧气等 ${ }^{[4]}$. 通过氧化反应产生的 $\mathrm{N}_{2} \mathrm{H}_{2}$ 分 子直接作为转移氢化试剂, 可以选择性地还原不饱和 $\mathrm{C}=\mathrm{C}$ 双键 ${ }^{[4]}$. 与经典的过渡金属催化的还原反应相比, 转移氢化反应为还原反应提供了另一种高效温和的还 原方法.

以水合肼 $\left(\mathrm{NH}_{2} \mathrm{NH}_{2} \cdot \mathrm{H}_{2} \mathrm{O}\right)$ 为转移氢化试剂, 通常是
在核黄素类化合物 ${ }^{[5]} 、$ 硒化合物 ${ }^{[6]}$ 和氧气 ${ }^{[7]}$ 等作为氧化剂 下转化成二氮烯 $\mathrm{N}_{2} \mathrm{H}_{2}$ 分子. 目前, 水合肼作为转移氢化 试剂的反应已经用于合成青蒿酸类化合物 ${ }^{[8]}$. 然而, 以 水合肼为转移氢化试剂还原不饱和 $\mathrm{C} \equiv \mathrm{C}$ 参键的研究较 少 ${ }^{[9 \sim 12]}$, 尤其是将不饱和 $\mathrm{C} \equiv \mathrm{C}$ 参键还原为不饱和 $\mathrm{C}=\mathrm{C}$ 双键的研究更少. 根据文献报道, Balci 等 ${ }^{[13]}$ 首次发现水 合肼可以将二苯乙炔分子还原为二苯乙烷和顺式-1,2二苯基乙烯分子, 但是顺式-1,2-二苯基乙烯分子的产率 较低, 二苯乙烷和顺式-1,2-二苯基乙烯分子的比例为 $86 ： 12$, 主要产物为完全还原的产物二苯乙烷，其反应 条件为: 高纯氧气作为氧化剂, 加入 4 倍量的水合肼, 反应时间为 $144 \mathrm{~h}$. 这是文献中首次报道的水合肼可以 将炔烃还原为烯烃的反应. 但是该反应存在的缺点是反 应时间长、产率低. 因此, 研究水合肼作为转移氢化试 剂快速、高效的选择性还原炔烃至烯烃的反应具有重要

\footnotetext{
* Corresponding authors. E-mail: kerangwang@hbu.edu.cn; lixl@hbu.edu.cn.

Received January 20, 2017; revised February 19, 2017; published online February 27, 2017.

Project supported by the National Natural Science Foundation of China (Nos. 21372059, 21572044), and the Natural Science Foundation of Hebei Province (Nos. B2016201254, 2016201031).

国家自然科学基金(Nos. 21372059, 21572044)和河北省自然科学基金(Nos. B2016201254, 2016201031)资助项目.
} 
的意义.

本文中, 我们在空气中以水合肼 $\left(\mathrm{NH}_{2} \mathrm{NH}_{2} \cdot \mathrm{H}_{2} \mathrm{O}\right)$ 为 转移氢化反应试剂, 研究了一系列具有空间位阻效应的 炔烃分子转化为烯烃化合物的反应. 研究发现, 水合肼 $\left(\mathrm{NH}_{2} \mathrm{NH}_{2} \cdot \mathrm{H}_{2} \mathrm{O}\right)$ 作为有效的转移氢化试剂, 在没有催化 剂的条件下, 在空气中高效地将炔烃还原至顺式烯烃. 通过 2D NOESY 谱研究了顺式烯烃的构型. 该研究结果 为以水合肼 $\left(\mathrm{NH}_{2} \mathrm{NH}_{2} \cdot \mathrm{H}_{2} \mathrm{O}\right)$ 为转移氢化反应试剂选择性 还原炔烃至烯烃的研究做了初步的尝试.

\section{1 结果与讨论}

研究水合肼还原炔烃至烯烃的反应源于通过盖布 瑞尔(Gabriel)合成法制备伯胺的反应. 众所周知, 水合 肼水解邻苯二甲酰亚胺类化合物是制备伯胺类化合物 常用的反应, 即盖布瑞尔合成法 ${ }^{[14]}$. 在我们前期的研究 工作中, 通过水合肼水解化合物 $\mathbf{1}$ 制备炔基修饰三甘醇 化合物 1a 时 ${ }^{[15]}$ (Eq. 1)发现制备的三甘醇衍生物是混合 物，含有炔基、烯基和烷基化合物 1a $\sim 1 \mathrm{c}$, 其中 $1 \mathrm{a} \sim 1 \mathrm{c}$ 的比例分别为 $22 \% 、 29 \%$ 和 $50 \%$, 混合物中烯烃化合物 $1 \mathrm{~b}$ 通过 ${ }^{1} \mathrm{H}$ NMR 谱确认, $\delta 5.89,5.26$ 和 5.15 的质子氢为 烯烃特征氢, 进一步通过 HRMS 谱确认了三种化合物
$1 \mathrm{a} \sim 1 \mathrm{c}$ 的分子量. 三种化合物 $1 \mathrm{a} \sim 1 \mathrm{c}$ 的含量通过 ${ }^{1} \mathrm{H}$ NMR 谱中的亚甲基质子氢的比例确认，其比例为 1 : $1.29 ： 2.24$. 该结果表明水合肼在水解邻苯二甲酰亚胺 的过程中将炔基还原成了烯基和烷基，说明水合肼 $\left(\mathrm{NH}_{2} \mathrm{NH}_{2} \cdot \mathrm{H}_{2} \mathrm{O}\right)$ 在空气中可作为转移氢化试剂.

进而, 我们研究了炔基修饰三甘醇化合物 $\mathbf{2}$ 的转移 氢化反应. 如表 1 所示, 在乙醇溶剂中, 16 倍量的质量 分数为 $80 \%$ 的水合肼，反应时间为 $4 \mathrm{~h}$, 随着温度升高， 烯烃衍生物 2a 的含量逐渐增加(表 1, Entries 1 6), 并 且完全还原的产物 $\mathbf{2 b}$ 的含量也增加. 在乙醇回流的条 件下，炔基化合物转化为烯烃化合物 $\mathbf{2 a}$ 和烷基化合物 2b 的含量分别为 $26 \%$ 和 $44 \%$, 不同组分化合物的含量 通过 ${ }^{1} \mathrm{H}$ NMR 谱确认. 进一步优化反应条件, 改变反应 时间, 发现随着反应时间的延长, 完全还原的产物 $\mathbf{2 b}$ 的 含量逐渐增加，当反应时间为 $24 \mathrm{~h}$ 时，炔基化合物 2 全 部转化为烷基衍生物 2b (表 1, Entries 7 12). 进而, 改 变水合肼的加入当量, 发现 16 倍量时, 化合物 $\mathbf{2}$ 的转化 率较高并且烯烃化合物 2a 的含量较高(表 1, Entries 13 15 ). 另外，将端炔化合物变为中间炔基化合物 $\mathbf{3}$, 反应 $12 \mathrm{~h}$ 只有 $13 \%$ 的炔基化合物 $\mathbf{3}$ 转化为烯烃化合物 $\mathbf{3 a}$ (表 2).

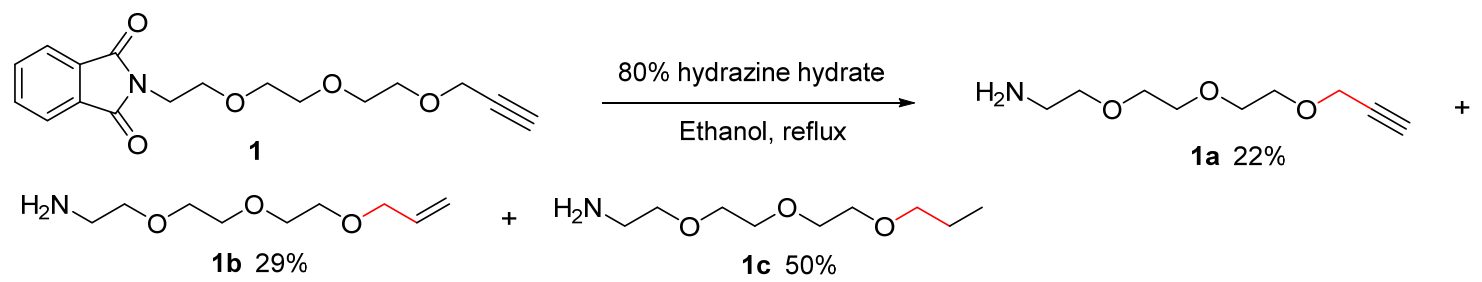

表 1 化合物 2 在不同反应温度、反应时间和水合肼当量条件下的反应结果

Table 1 Temperature, time and equivalent effects on the reduction of compound 2

\begin{tabular}{|c|c|c|c|c|c|}
\hline Entry & $t / \mathrm{h}$ & $T /{ }^{\circ} \mathrm{C}$ & $\mathrm{NH}_{2} \mathrm{NH}_{2} \cdot \mathrm{H}_{2} \mathrm{O}$ /equiv. & $2 / 2 a / 2 b^{a}$ & Conv. ${ }^{a} / \%$ of $\mathbf{2 a} / \mathbf{2 b}$ \\
\hline 1 & 4 & 20 & 16 & $1 / 0.03 / 0$ & $2.9 / 0$ \\
\hline 2 & 4 & 40 & 16 & $1 / 0.06 / 0$ & $5.8 / 0$ \\
\hline 3 & 4 & 50 & 16 & $1 / 0.2 / 0.03$ & $16 / 2.4$ \\
\hline 4 & 4 & 60 & 16 & $1 / 0.44 / 0.16$ & $28 / 10$ \\
\hline 5 & 4 & 70 & 16 & $1 / 0.74 / 0.70$ & $30 / 29$ \\
\hline 6 & 4 & Reflux & 16 & $1 / 0.85 / 1.48$ & $26 / 44$ \\
\hline 7 & 2 & Reflux & 16 & $1 / 0.44 / 0.19$ & $27 / 12$ \\
\hline 8 & 4 & Reflux & 16 & $1 / 0.85 / 1.48$ & $26 / 44$ \\
\hline 9 & 6 & Reflux & 16 & $1 / 1.21 / 2.53$ & $26 / 53$ \\
\hline 10 & 8 & Reflux & 16 & $1 / 1.44 / 3.99$ & $22 / 62$ \\
\hline 11 & 12 & Reflux & 16 & $1 / 1.79 / 9.35$ & $15 / 77$ \\
\hline 12 & 24 & Reflux & 16 & $0 / 0 / 100$ & $0 / 100$ \\
\hline 13 & 4 & Reflux & 2 & $1 / 0.18 / 0.02$ & $15 / 1.7$ \\
\hline 14 & 4 & Reflux & 8 & $1 / 0.48 / 0.19$ & $29 / 11$ \\
\hline 15 & 4 & Reflux & 16 & $1 / 0.85 / 1.48$ & $26 / 44$ \\
\hline
\end{tabular}

${ }^{a}$ Determined by ${ }^{1} \mathrm{H}$ NMR spectroscopy. 
表 2 化合物 3 在不同反应时间下的反应结果 ${ }^{a}$

Table 2 Time effects on the reduction of compound 3<smiles>CC#CCOCCOCCOCCO</smiles><smiles>C/C=C\COCCOCCOCCO</smiles>

$\mathrm{HO}_{\mathrm{O}} \mathrm{NO}_{\mathrm{O}}$

3b

\begin{tabular}{cccc}
\hline Entry & Time $/ \mathbf{h}$ & $\mathbf{3} / \mathbf{3 a} / \mathbf{3} \mathbf{b}^{a}$ & Conversion $^{b} / \%$ of $\mathbf{3 a} / \mathbf{3 b}$ \\
\hline 1 & 4 & $1 / 0.08 / 0.01$ & $7.3 / 0.9$ \\
2 & 6 & $1 / 0.12 / 0.03$ & $10.4 / 2.6$ \\
3 & 8 & $1 / 0.11 / 0.01$ & $9.8 / 0.8$ \\
4 & 12 & $1 / 0.16 / 0.04$ & $13.3 / 3.3$ \\
\hline
\end{tabular}

${ }^{a}$ Reaction temperature: reflux; $\mathrm{NH}_{2} \mathrm{NH}_{2} \cdot \mathrm{H}_{2} \mathrm{O}: 16$ equiv. ${ }^{b}$ Determined by ${ }^{1} \mathrm{H}$ NMR spectroscopy.

通过上述研究结果可知, 水合肼在空气中可以作为 转移氢化试剂还原炔烃, 并且能制备其中间还原产物烯 烃, 然而, 转化率较低, 并有过度还原的烷烃产物.

因此, 我们改变反应底物的结构, 研究了一系列双 烷基和羟基取代的炔烃化合物 4 7 的还原反应，如 Eq. 2 所示, 反应底物的炔基两端都具有一定的空间位阻效 应.<smiles>[R]C([Y])(O)C#CC([Z])(C)O</smiles>
$\mathrm{R}=\mathrm{CH}_{3} 4, \mathrm{CH}_{2} \mathrm{CH}_{3} 5, \mathrm{CH}_{2} \mathrm{CH}_{2} \mathrm{CH}_{3} 6, \mathrm{CH}_{2} \mathrm{CH}\left(\mathrm{CH}_{3}\right)_{2} 7$

化合物 2,5-二甲基-3-炔-2,5-己二醇(4)在乙醇溶液 中，16 倍量的 $80 \%$ 水合肼，回流条件下，随着反应时间 从 $1 \mathrm{~h}$ 到 $4 \mathrm{~h}$, 烯烃化合物 $\mathbf{4 a}$ 的反应转化率分别为 $58 \%$ (1 h)、69\% (2 h)、89 (3 h) 和 89\% (4 h)(表 3). 当反应时 间为 $4 \mathrm{~h}$ 时, 完全还原成烷烃化合物 $\mathbf{4 b}$ 的转化率为 $3.5 \%$, 说明反应时间为 $3 \mathrm{~h}$ 为最优的反应时间, 由炔烃 底物转化为烯烃化合物 $4 \mathrm{a}$ 的转化率达到 $89 \%$. 烯烃衍 生物 $4 \mathbf{a}$ 经柱层析分离, 洗脱剂为 $\mathrm{CH}_{2} \mathrm{Cl}_{2} / \mathrm{CH}_{3} \mathrm{COOEt}$ $(V / V=3 / 1)$, 化合物结构经 ${ }^{1} \mathrm{H} \mathrm{NMR} 、{ }^{13} \mathrm{C} \mathrm{NMR}$ 和 HRMS 等表征.

进而, 研究了空间位阻效应与反应转化率的关系, 分别以 3,6-二甲基-4-炔-3,6-辛二醇(5)、4,7-二甲基-5炔-4,7-癸二醇(6)和 2,4,7,9-四甲基-5-炔-4,7-癸二醇(7)为 反应底物, 在乙醇溶剂中, 16 倍当量的 $80 \%$ 水合肼条件 下，在不同反应时间转化为烯烃化合物 5a、6a 和 7a(表 4). 研究结果表明, 制备的烯烃化合物 5a、6a 和 7a 的 转化率分别为 $92 \% 、 90 \%$ 和 94\%(表 4), 说明随着空间位 阻的增大, 由炔烃转化为烯烃衍生物的转化率升高. 另 外, 由于反应底物的空间位阻增大, 反应时间也从化合 物 $4 \mathbf{a}$ 的 $3 \mathrm{~h}$ 延长到 21 (5a)、24 (6a) 和 $26 \mathrm{~h}$ (7a). 烯烃衍
表 3 化合物 4 在不同反应时间下的反应结果

Table 3 Times effects on the reduction of compound 4


$4 a$

$4 b$

\begin{tabular}{cccc}
\hline Entry & $t / \mathbf{h}$ & $\mathbf{4} / \mathbf{4} \mathbf{a} / \mathbf{4} \mathbf{b}^{a}$ & Conv. $^{a} / \%$ of $\mathbf{4 a} / \mathbf{4 b}$ \\
\hline 1 & 1 & $0.74 / 1 / 0$ & $58 / 0$ \\
2 & 2 & $0.46 / 1 / 0$ & $69 / 0$ \\
3 & 3 & $0.13 / 1 / 0$ & $89 / 0$ \\
4 & 4 & $0.09 / 1 / 0.04$ & $89 / 3.5$ \\
\hline
\end{tabular}

${ }^{a}$ Determined by ${ }^{1} \mathrm{H}$ NMR spectroscopy.

生物 $5 \mathbf{a} 、 6 \mathbf{a}$ 和 $7 \mathbf{a}$ 经柱层析分离, 洗脱剂为 $\mathrm{CH}_{2} \mathrm{Cl}_{2}$ / $\mathrm{CH}_{3}$ COOEt $(V / V=3 / 1)$, 化合物结构经 ${ }^{1} \mathrm{H} \mathrm{NMR} 、{ }^{13} \mathrm{C}$ NMR 和 HRMS 等表征.

表 4 化合物 5 7 在不同反应时间下的反应结果

Table 4 Reduction of compounds 5, 6 and 7 with different reaction times
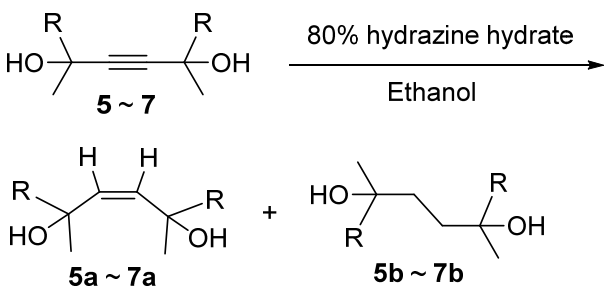

\begin{tabular}{|c|c|c|c|c|}
\hline Alkyne & $\mathrm{R}$ & $t / \mathrm{h}$ & $\begin{array}{c}\text { Alkyne/alkene/ } \\
\text { alkane }^{a}\end{array}$ & Conv. ${ }^{a} / \%$ of $\mathbf{a} / \mathbf{b}$ \\
\hline \multirow{7}{*}{5} & \multirow{7}{*}{$\mathrm{CH}_{3} \mathrm{CH}_{2}$} & 2 & $1.3 / 1 / 0$ & $44 / 0$ \\
\hline & & 4 & $0.73 / 1 / 0$ & $59 / 0$ \\
\hline & & 6 & $0.44 / 1 / 0$ & $69 / 0$ \\
\hline & & 8 & $0.30 / 1 / 0.02$ & $76 / 1.5$ \\
\hline & & 12 & $0.11 / 1 / 0.03$ & $88 / 2.6$ \\
\hline & & 17 & $0.07 / 1 / 0.04$ & $90 / 3.6$ \\
\hline & & 21 & $0.02 / 1 / 0.07$ & $92 / 6.4$ \\
\hline \multirow{4}{*}{6} & \multirow{4}{*}{$\mathrm{CH}_{3} \mathrm{CH}_{2} \mathrm{CH}_{2}$} & 4 & $0.58 / 1 / 0$ & $63 / 0$ \\
\hline & & 8 & $0.52 / 1 / 0$ & $66 / 0$ \\
\hline & & 24 & $0 / 1 / 0.11$ & $90 / 9.9$ \\
\hline & & 48 & $0 / 1 / 0.23$ & $81 / 18$ \\
\hline \multirow{4}{*}{7} & \multirow{4}{*}{$\left(\mathrm{CH}_{3}\right)_{2} \mathrm{CHCH}_{2}$} & 2 & $3.82 / 1 / 0$ & $21 / 0$ \\
\hline & & 12 & $0.53 / 1 / 0$ & $65 / 0$ \\
\hline & & 24 & $0.05 / 1 / 0.03$ & $93 / 2.8$ \\
\hline & & 26 & $0 / 1 / 0.06$ & $94 / 5.7$ \\
\hline
\end{tabular}

${ }^{a}$ Determined by ${ }^{1} \mathrm{H}$ NMR spectroscopy.

进而研究了烯烃化合物的构型，(Z)-2,5-二甲基-3烯-2,5-辛二醇 $(\mathbf{5 a})$ 的 ${ }^{1} \mathrm{H} \mathrm{NMR}$ 和 ${ }^{13} \mathrm{C} \mathrm{NMR}$ 与文献报道的 结果一致 ${ }^{[16]}$. 另外我们研究了不对称炔烃化合物 2-甲 基-4-苯基-3-炔-2-丁醇(8)选择性还原为烯烃的反应(Eq. $3)$, 发现反应 12 和 $24 \mathrm{~h}$ 生成的烯烃化合物 $8 \mathrm{a}$ 的转化率 
只有 $26 \%$ 和 $25 \%$, 随着反应时间延长，“完全” 还原的 产物 $8 \mathrm{~b}$ 的量增加, 说明芳基的引入降低了炔烃的反应 活性和反应的选择性.

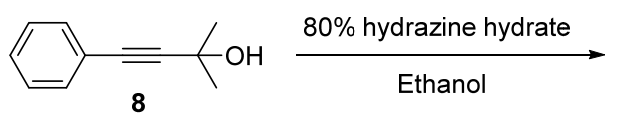<smiles>CC(C)(O)CCc1ccccc1</smiles>

烯烃化合物 8a 经柱层析分离, ${ }^{1} \mathrm{H} N M R$ 和 ${ }^{13} \mathrm{C} N M R$ 谱表征. 烯烃化合物 8a 的 ${ }^{1} \mathrm{H} N \mathrm{NR}$ 谱显示 $\mathrm{C}=\mathrm{C}$ 双键的 质子氢分别在 $\delta 6.47$ 和 5.77 , 偶合常数为 $12.6 \mathrm{~Hz}$, 说明 其为顺式烯烃的结构. 进而, 2D NOESY 谱研究了化合 物 8a 的构型, 苯基的特征质子氢( $\delta 7.23$ 和 7.32)与甲基 的质子氢( $\delta$ 1.36)和羟基的质子氢( $(\delta 1.57)$ 之间存在明显 的相关, 如图 1 所示. 另外, 双键的特征质子氢 ( $\delta$ 5.77) 与甲基的质子氢 $(\delta$ 1.57)也存在明显的相关, 如图 1 所 示. 这些结果说明化合物 $8 \mathbf{a}$ 是顺式结构.

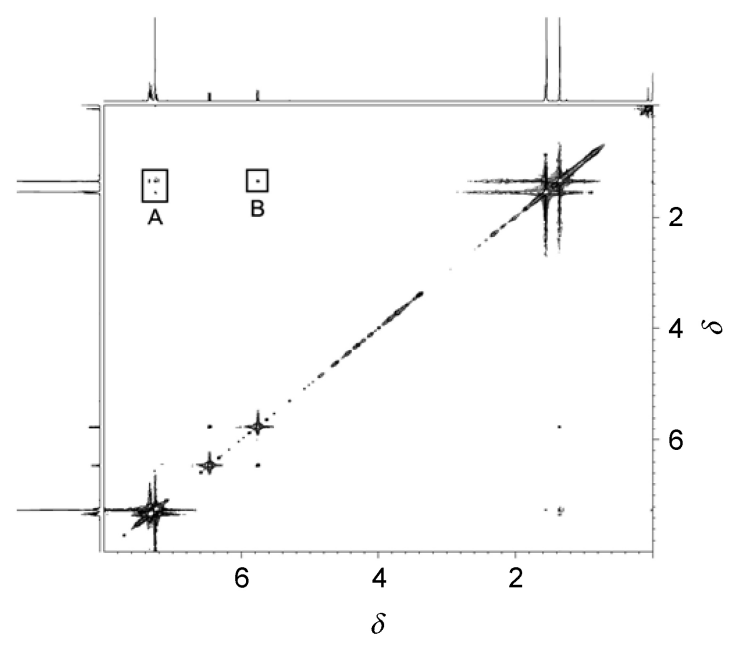

图 1 化合物 8a 的 2D NOESY 谱

Figure 1 2D NOESY spectrum of compound $\mathbf{8 a}$

水合肼选择性还原炔烃至顺式烯烃的反应机理 (Scheme 1) 与文献[4]报道的水合肼将烯烃还原为烷烃的 机理类似, 即水合肼在空气中氧化生成二氮烯 $\mathrm{N}_{2} \mathrm{H}_{2}$ 分 子 ${ }^{[7]}$, 进而二氮烯 $\mathrm{N}_{2} \mathrm{H}_{2}$ 分子与炔烃底物分子形成六元环 状过渡态, 氢原子与炔键顺式加成, 氢原子转移到炔键 上. 由于炔烃底物分子的空间位阻效应, 降低了生成的 烯烃化合物的反应活性, 最终产物保留在顺式烯烃阶 段.

\section{2 结论}

研究了一系列双烷基和双羟基修饰的炔类化合物,

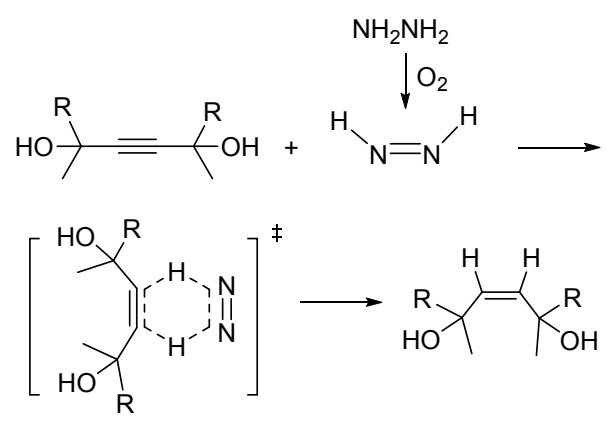

图式 1 水合肼作为转移氢化试剂发生还原反应的机理 Scheme 1 Oxidation of hydrazine to diazene in the air and transfer hydraogenation of alkynes to alkenes

在以水合肼 $\left(\mathrm{NH}_{2} \mathrm{NH}_{2} \cdot \mathrm{H}_{2} \mathrm{O}\right)$ 为转移氢化试剂的条件下, 在空气中高效地将炔烃还原为顺式烯烃的反应. 反应机 理为水合肼在空气中氧化生成二氮烯 $\mathrm{N}_{2} \mathrm{H}_{2}$ 分子, 二氮 烯 $\mathrm{N}_{2} \mathrm{H}_{2}$ 分子与炔烃底物分子形成六元环状过渡态, 发 生顺式加成，由于底物分子取代基的空间位阻效应，降 低了生成的烯烃化合物的反应活性，最终产物保留在顺 式烯烃阶段. 进而, 通过 2D NOESY 谱证明了生成的不 对称的烯烃化合物的构型为顺式构型. 该研究结果为以 水合肼为转移氢化试剂选择性将炔烃还原至烯烃的研 究做了初步尝试.

\section{3 实验部分}

\section{1 仪器与试剂}

${ }^{1} \mathrm{H}$ NMR、 ${ }^{13} \mathrm{C}$ NMR 和 2D NOESY 谱用 RT-NMR Bruker AVANCE $600 \mathrm{MHz}$ (TMS 为内标)测量; 高分辨 率质谱用 FTICR-MD (Ionspec 7.0) 测定.

\section{2 实验方法}

\subsection{1 (Z)-2,5-二甲基-3-烯-2,5-已二醇 $(4 \mathbf{a})$ 的合成}

$25 \mathrm{~mL}$ 圆底烧瓶中, 加入 $710 \mathrm{mg}(5.0 \mathrm{mmol})$ 的 2,5二甲基-3-炔-2,5-己二醇(4), $4.9 \mathrm{~mL}$ (80 mmol)的水合肼 $\left(\mathrm{NH}_{2} \mathrm{NH}_{2} \cdot \mathrm{H}_{2} \mathrm{O}\right)(80 \%)$, 加入 $20 \mathrm{~mL}$ 乙醇为溶剂. 该反应 体系在空气中回流 $3 \mathrm{~h}$, 停止反应. 反应液冷至室温, 旋 干溶剂, 剩余物溶解在 $10 \mathrm{~mL}$ 二氯甲烷溶剂中, 用 $5 \mathrm{~mL}$ 水洗两次，除去剩余的水合肼，有机相用无水硫酸钠干 燥. 柱层析分离, 洗脱剂为 $\mathrm{CH}_{2} \mathrm{Cl}_{2} / \mathrm{CH}_{3} \mathrm{COOEt}(V / V=$ $3 / 1)$, 得到白色固体化合物 $\mathbf{4 a}(628 \mathrm{mg})$, 产率 87\%. m.p. $68.2 \sim 68.8{ }^{\circ} \mathrm{C} ;{ }^{1} \mathrm{H}$ NMR (600 $\left.\mathrm{MHz}, \mathrm{CDCl}_{3}\right) \delta: 1.39$ (s, $\left.12 \mathrm{H}, \mathrm{CH}_{3}\right), 4.25(\mathrm{~s}, 2 \mathrm{H}, \mathrm{OH}), 5.34(\mathrm{~s}, 2 \mathrm{H}, \mathrm{C}=\mathrm{CH}) ;{ }^{13} \mathrm{C}$ NMR (150 MHz, $\left.\mathrm{CDCl}_{3}\right) \delta: 31.59\left(\mathrm{CH}_{3}\right), 71.14(\mathrm{C}-\mathrm{C}=$ C), $135.41(\mathrm{C}=\mathrm{C})$; HRMS calcd for $\mathrm{C}_{8} \mathrm{H}_{16} \mathrm{NaO}_{2}$ 167.1048, found 167.1043 .

3.2 .2 合成化合物 $5 \mathbf{a} 、 \mathbf{6 a}$ 和 $7 \mathbf{a}$ 的方法

在 $25 \mathrm{~mL}$ 圆底烧瓶中, 加入 $5.0 \mathrm{mmol}$ 的化合物 $\mathbf{5} \sim$ 
7, $4.9 \mathrm{~mL}$ (80 mmol)的质量分数 $80 \%$ 水合肼 $\left(\mathrm{NH}_{2} \mathrm{NH}_{2}\right.$ • $\mathrm{H}_{2} \mathrm{O}$ ), 加入 $20 \mathrm{~mL}$ 乙醇为溶剂. 反应时间为 $21 \sim 26 \mathrm{~h}$, 停止反应, 反应液冷至室温, 旋干溶剂, 剩余物溶解在 $10 \mathrm{~mL}$ 二氯甲烷溶剂中, 用 $5 \mathrm{~mL}$ 水洗两次, 除去剩余的 水合肼, 有机相用无水硫酸钠干燥. 柱层析分离, 洗脱 剂为 $\mathrm{CH}_{2} \mathrm{Cl}_{2} / \mathrm{CH}_{3} \mathrm{COOEt}(V / V=3 / 1)$, 制备化合物 $\mathbf{5 a} \sim$ $7 \mathbf{a}$.

(Z)-3,6-二甲基-4-烯-3,6-辛二醇 $(\mathbf{5 a})$ ：产率 90\%. 粘 稠液体. ${ }^{1} \mathrm{H}$ NMR $\left(600 \mathrm{MHz}, \mathrm{CDCl}_{3}\right) \delta: 0.91 \sim 0.94(\mathrm{~m}$, $\left.6 \mathrm{H}, \mathrm{CH}_{3}\right), 1.33\left(\mathrm{~d}, J=4.2 \mathrm{~Hz}, 6 \mathrm{H}, \mathrm{CH}_{3}\right), 1.59 \sim 1.63(\mathrm{~m}$, $\left.4 \mathrm{H}, \mathrm{CH}_{2}\right), 4.13(\mathrm{~s}, 2 \mathrm{H}), 5.21(\mathrm{~s}, 2 \mathrm{H}, \mathrm{C}=\mathrm{CH}) ;{ }^{13} \mathrm{C} \mathrm{NMR}$ $\left(150 \mathrm{MHz}, \mathrm{CDCl}_{3}\right) \delta: 8.55\left(\mathrm{CH}_{3}\right), 29.26,29.34,36.46$, $36.55,73.44,135.06,135.12$; HRMS calcd for $\mathrm{C}_{10} \mathrm{H}_{20} \mathrm{NaO}_{2}$ $[\mathrm{M}+\mathrm{Na}]^{+}:$195.1361, found 195.1353 .

(Z)-4,7-二甲基-5-烯-4,7-癸二醇(6a)：产率 $88 \%$. 粘 稠液体. ${ }^{1} \mathrm{H} \mathrm{NMR}\left(600 \mathrm{MHz}, \mathrm{CDCl}_{3}\right) \delta: 0.92(\mathrm{t}, J=7.2 \mathrm{~Hz}$, $\left.6 \mathrm{H}, \mathrm{CH}_{3}\right), 1.34\left(\mathrm{~d}, J=3.6 \mathrm{~Hz}, 6 \mathrm{H}, \mathrm{CH}_{3}\right), 1.33 \sim 1.43(\mathrm{~m}$, $\left.4 \mathrm{H}, \mathrm{CH}_{2}\right), 1.54 \sim 1.58\left(\mathrm{~m}, 4 \mathrm{H}, \mathrm{CH}_{2}\right), 4.06(\mathrm{~s}, 2 \mathrm{H}, \mathrm{OH})$, $5.30(\mathrm{~d}, J=1.8 \mathrm{~Hz}, 2 \mathrm{H}, \mathrm{C}=\mathrm{CH}) ;{ }^{13} \mathrm{C} \mathrm{NMR}(150 \mathrm{MHz}$, $\left.\mathrm{CDCl}_{3}\right) \delta: 14.48,17.50,29.75,29.81,46.39,46.47,73.23$, 135.05, 135.11; HRMS calcd for $\mathrm{C}_{12} \mathrm{H}_{25} \mathrm{O}_{2}[\mathrm{M}+\mathrm{H}]^{+}$: 201.1855, found 201.1851.

(Z)-2,4,7,9-四甲基-5-烯-4,7-癸二醇(7a): 产率 94\%. m.p. 52.2 53.8 ${ }^{\circ} \mathrm{C} ;{ }^{1} \mathrm{H}$ NMR $\left(600 \mathrm{MHz}, \mathrm{CDCl}_{3}\right): 0.95 \sim$ $0.97\left(\mathrm{~m}, 12 \mathrm{H}, \mathrm{CH}_{3}\right), 1.35\left(\mathrm{~s}, 6 \mathrm{H}, \mathrm{CH}_{3}\right), 1.51(\mathrm{~d}, J=6.0 \mathrm{~Hz}$, $\left.4 \mathrm{H}, \mathrm{CH}_{2}\right), 1.83 \sim 1.87(\mathrm{~m}, 2 \mathrm{H}, \mathrm{CH}), 4.06(\mathrm{~s}, 2 \mathrm{H}, \mathrm{OH}), 5.32$ $(\mathrm{s}, 2 \mathrm{H}, \mathrm{C}=\mathrm{CH}) ;{ }^{13} \mathrm{C} \mathrm{NMR}\left(150 \mathrm{MHz}, \mathrm{CDCl}_{3}\right) \delta: 24.54$, 24.62, 24.69, 30.80, 52.73, 73.93, 135.33; HRMS calcd for $\mathrm{C}_{14} \mathrm{H}_{28} \mathrm{NaO}_{2}[\mathrm{M}+\mathrm{Na}]^{+}: 251.1987$, found 251.1985.

\section{2 .3 化合物 $8 \mathbf{a}$ 的合成}

在 $25 \mathrm{~mL}$ 圆底烧瓶中, 加入 $800 \mathrm{mg}(5.0 \mathrm{mmol})$ 的 2甲基-4-苯基-3-炔- 2-丁醇 $(8), 4.9 \mathrm{~mL}$ (80 mmol)的水合肼 $\left(\mathrm{NH}_{2} \mathrm{NH}_{2} \cdot \mathrm{H}_{2} \mathrm{O}\right)(80 \%)$, 加入 $20 \mathrm{~mL}$ 乙醇为溶剂, 在空气 中反应 $12 \mathrm{~h}$. 反应液冷至室温, 旋干溶剂, 剩余物溶解 在 $10 \mathrm{~mL}$ 二氯甲烷溶剂中, 用 $5 \mathrm{~mL}$ 水洗两次, 除去剩余 的水合肼, 有机相用无水硫酸钠干燥. 柱层析分离, 洗 脱剂为 $\mathrm{CH}_{2} \mathrm{Cl}_{2} / \mathrm{CH}_{3} \mathrm{COOEt}(V / V=3 / 2)$, 得白色固体 (Z)-2-甲基-4-苯基-3-烯-2-丁醇(8a) (225.8 mg), 产率 25\%. m.p. 57.5 58.2 ${ }^{\circ} \mathrm{C} ;{ }^{1} \mathrm{H}$ NMR $\left(\mathrm{CDCl}_{3}, 600 \mathrm{MHz}\right) \delta$ : $1.36\left(\mathrm{~s}, 6 \mathrm{H}, \mathrm{CH}_{3}\right), 1.57(\mathrm{~s}, 1 \mathrm{H}, \mathrm{OH}), 5.77$ (d, 1H, $J=12.6$ $\mathrm{Hz}, \mathrm{CH}), 6.47$ (d, 1H, $J=12.6 \mathrm{~Hz}, \mathrm{CH}), 7.23$ (t, 1H, $J=7.2$ $\mathrm{Hz}, \mathrm{ArH}), 7.31 \sim 7.35(4 \mathrm{H}, \mathrm{ArH}) ;{ }^{13} \mathrm{C} \mathrm{NMR}\left(\mathrm{CDCl}_{3}, 150\right.$ $\mathrm{MHz}) \delta$ : 31.20, 72.12, 126.98, 127.89, 128.08, 129.01, 137.55, 139.34. Anal. calcd for $\mathrm{C}_{11} \mathrm{H}_{14} \mathrm{O}: \mathrm{C} 81.44, \mathrm{H} 8.70$; found C 81.32, H 8.79.
辅助材料(Supporting Information) 化合物的核磁共 振谱、高分辨率质谱等数据. 这些材料可以免费从本刊 网站(http://sioc-journal.cn/)上下载.

\section{Referenes}

[1] (a) de Vries, J. G.; Elsevier, C. J. The Handbook of Homogeneous Hydrogenation. Wiley-VCH, Weinheim, 2007.

(b) Chernichenko, K.; Madarász, Á.; Pápai, I.; Nieger, M.; Leskelä, M.; Repo, T. Nat. Chem. 2013, 5, 718.

[2] (a) Johnstone, R. A. W.; Wilby, A. H.; Entwistle, I. D. Chem. Rev. $\mathbf{1 9 8 5}, 85,129$.

(b) Hauwert, P.; Maestri, G.; Sprengers, J. W.; Catellani, M.; Elsevier, C. J. Angew. Chem. Int. Ed. 2008, 47, 3223.

(c) Hauwert, P.; Boerleider, R.; Waesink, S.; Weigand, J. J.; Elsevier, C. J. J. Am. Chem. Soc. 2010, 132, 16900.

(d) Heim, L. E.; Thiel, D.; Gedig, C.; Deska, J.; Prechtl, M. H. G. Angew. Chem., Int. Ed. 2015, 54, 10308.

(e) Yang, X. H.; Xie, Z. M.; He, J.; Yu, L. Chin. J. Org. Chem. 2015, 35, 603 (in Chinese).

(阳香华, 谢珍苔, 何军, 余林, 有机化学, 2015, 35, 603.)

(f) Dai, N.; Shang, R.; Fu, M. C.; Fu, Y. Chin. J. Chem. 2015, 33, 405.

[3] Wang, D.; Astruc, D. Chem. Rev. 2015, 115, 6621.

[4] (a) Hünig, S.; Müller, H. R.; Thier, W. Angew. Chem., Int. Ed. Engl. $\mathbf{1 9 6 5}, 4,271$

(b) Pasto, D. J. Org. Rect. 1991, 40, 91.

(c) Gutmann, B.; Cantillo, D.; Kappe, C. O. Angew. Chem., Int. Ed. 2015, 54, 6688 .

[5] (a) Imada, Y.; Iida, H.; Naota, T. J. Am. Chem. Soc. 2005, 127, 14544.

(b) Teichert, J. F.; den Hartog, T.; Hanstein, M.; Smit, C.; ter Horst, B.; Hernandez-Olmos, V.; Feringa, B. L.; Minnaard, A. J. ACS Catal. 2011, 1, 309.

(c) Lida, H.; Imada, Y.; Murahashi, S. I. Org. Biomol. Chem. 2015, 13, 7599 .

[6] Schulz, G. A. S.; Comin, E.; de Souza, R. F. J. Appl. Polym. Sci. 2012, 123, 3605 .

[7] (a) Pieber, B.; Martinez, S. T.; Cantillo, D.; Kappe, C. O. Angew Chem., Int. Ed. 2013, 52, 10241.

(b) Santra, S.; Guin, J. Eur. J. Org. Chem. 2015, 7253.

[8] (a) Feth, M. P.; Rossen, K.; Burgard, A. Org. Process Res. Dev. 2013, 17, 282.

(b) Pieber, B.; Glasnov, T.; Kappe, C. O. Chem.-Eur. J. 2015, 21, 4368.

[9] Smit, C.; Fraaije, M. W.; Minnaard, A. J. J. Org. Chem. 2008, 73, 9482.

[10] Imada, Y.; Iida, H.; Kitagawa, T.; Naota, T. Chem.-Eur. J. 2011, 17, 5908.

[11] Lamani, M.; Ravikumara, G. S.; Prabhu, K. R. Adv. Synth. Catal. 2012, 354, 1437.

[12] Lamani, M.; Guralamata, R. S.; Prabhu, K. R. Chem. Commun. 2012, 48, 6583.

[13] Menges, N.; Balci, M. Synlett 2014, 25, 671.

[14] (a) Yu, T. B.; Bai, J. Z.; Guan, Z. Angew. Chem., Int. Ed. 2009, 48, 1097.

(b) Stockdill, J. L.; lopez, A. M.; Ibrahim, A. A. Tetrahedron Lett. 2015, 56, 3503.

(c) Hua, J.; Lam, J. W. Y.; Yu, X. M.; Wu, L. J.; Kwok, H. S.; Wong, K. S.; Tang, B. Z. J. Polym. Sci: Pol. Chem. 2008, 46, 2025.

[15] Wang, K. R.; Yang, Z. B.; Li, X. L. Chem.-Eur. J. 2015, 21, 5680.

[16] Sajiki, H.; Mori, S.; Ohkubo, T.; Ikawa, T.; Kume, A.; Maegawa, T.; Monguchi, Y. Chem.-Eur. J. 2008, 14, 5109.

(Li, L.; Fan, Y.) 